\title{
Efecto de la suplementación energético-proteica sobre el desempeño productivo en vacas Romosinuano durante el pre y posparto
}

\author{
Effect of energy-protein supplementation on productive performance of \\ Romosinuano cows during pre and postpartum
}

\author{
Lorena-Inés Mestra Vargas ${ }^{1,4}$, Rómulo Campos Gaona ${ }^{2}$, Natalia Herrera Perez ${ }^{1}$, \\ Juan Carlos Fernández Niño ${ }^{1}$, Katherine García Alegría ${ }^{3}$
}

\section{Resumen}

Se evaluó el efecto de densidades energéticas y proteicas de dietas sobre la homeostasis, perfil metabólico y desempeño productivo durante el pre y postparto de vacas Romosinuano. Treinta vacas en pastoreo fueron asignadas a tres grupos de suplementación nutricional: TC: 0.0 T1: 2.35 y T2: $2.8 \mathrm{~kg} /$ animal/día de suplemento energético-proteico. El experimento se desarrolló desde 30 días antes del parto hasta 60 días posparto. Se evaluó el peso corporal (Pcorp), condición corporal (CC) y concentraciones séricas de glucosa, colesterol, $\beta$-hidroxibutirato, ácidos grasos no esterificados (NEFAs), proteína total, urea, aspartato aminotransferasa (AST) y alanina aminotransferasa (ALT). La suplementación al preparto no afectó el Pcorp $(\mathrm{p}=0.4557)$ y CC $(\mathrm{p}=0.1893)$. En el posparto hubo efecto significativo de la suplementación sobre PC $(\mathrm{p}=0.0343)$ y CC $(p=0.029)$ en T1 y T2 y de glucosa al preparto en T2 $(p=0.0019)$. Para colesterol hubo efecto significativo en el preparto en $\mathrm{T} 2(\mathrm{p}=0.0036)$, así como al día 5 y 60 del posparto en

${ }^{1}$ Corporación Colombiana de Investigación Agropecuaria-AGROSAVIA; Centro de Investigación Turipaná, Córdoba, Colombia

${ }^{2}$ Departamento de Ciencia Animal, Universidad Nacional de Colombia, Palmira, Valle del Cauca, Colombia

${ }^{3}$ Servicio Nacional de Aprendizaje SENA, Buga, Valle del Cauca, Colombia

${ }^{4}$ Email: lmestra@agrosavia.co

Recibido; 12 de abril de 2021

Aceptado para publicación: 15 de noviembre de 2021

Publicado: 22 de diciembre de 2021

CLos autores. Este artículo es publicado por la Rev Inv Vet Perú de la Facultad de Medicina Veterinaria, Universidad Nacional Mayor de San Marcos. Este es un artículo de acceso abierto, distribuido bajo los términos de la licencia Creative Commons Atribución 4.0 Internacional (CC BY 4.0) [https:// creativecommons.org/licenses/by/4.0/deed.es] que permite el uso, distribución y reproducción en cualquier medio, siempre que la obra original sea debidamente citada de su fuente original 
T1 y en T2 ( $\mathrm{p}=0.001)$. En $\beta$-hidroxibutirato hubo efecto significativo a los días 5 y 12 posparto en TC $(\mathrm{p}=0.0365)$. Para NEFAs no hubo diferencias entre tratamientos en las dos fases. Para proteína total se encontró efecto significativo en el posparto en T2 $(\mathrm{p}=0.0163)$, en tanto que para urea fue en el preparto en TC $(\mathrm{p}=0.0334)$. Para AST hubo efecto significativo al día 5 del posparto en T2 $(\mathrm{p}=0.0202)$ y para ALT al día 60 posparto en T2 ( $\mathrm{p}=0.0018)$. El suministro de hasta $2.8 \mathrm{~kg} /$ animal/día de suplemento energéticoproteico optimiza el estado metabólico de vacas Romosinuano durante el pre y postparto, y el desempeño productivo, Pcorp y CC al posparto.

Palabras clave: Romosinuano, nutrición, perfil metabólico, preparto, postparto

\section{AbSTRACT}

The effect of energy and protein densities of diets on homeostasis, metabolic profile and productive performance during pre and postpartum periods of Romosinuano cows was evaluated. Thirty grazing cows were assigned to three nutritional supplementation groups: TC: $0.0, \mathrm{~T} 1: 2.35$ and T2: $2.8 \mathrm{~kg} / \mathrm{animal} / \mathrm{day}$ of energy-protein supplement. The experiment ran from 30 days before calving to 60 days postpartum. Body weight (BW), body condition (BCS) and serum concentrations of glucose, cholesterol, total protein, $\beta$ hydroxybutyrate, non-esterified fatty acids (NEFAs), urea, aspartate aminotransferase (AST) and alanine aminotransferase (ALT) were evaluated. The prepartum supplementation did not affect $\mathrm{BW}(\mathrm{p}=0.4557)$ nor $\mathrm{BCS}(\mathrm{p}=0.1893)$. In the postpartum period, there was a significant effect of supplementation on BW $(\mathrm{p}=0.0343)$ and $\mathrm{BCS}$ $(p=0.029)$ in T1 and T2 and on glucose at prepartum in T2 $(p=0.0019)$. For cholesterol, there was a significant effect on prepartum in $\mathrm{T} 2(\mathrm{p}=0.0036)$, as well as on days 5 and 60 postpartum in $\mathrm{T} 1$ and $\mathrm{T} 2(\mathrm{p}=0.001)$. In $\beta$-hydroxybutyrate there was a significant effect on postpartum days 5 and 12 in TC ( $\mathrm{p}=0.0365)$. For NEFAs there were no differences between treatments in the two phases. For total protein, a significant effect was found in the postpartum in $\mathrm{T} 2(\mathrm{p}=0.0163)$ while for urea it was in the prepartum in TC $(\mathrm{p}=0.0334)$. For AST there was a significant effect on postpartum day 5 in T2 $(\mathrm{p}=0.0202)$ and for ALT at postpartum day 60 in T2 ( $\mathrm{p}=0.0018)$. The supply of up to $2.8 \mathrm{~kg} / \mathrm{animal} /$ day of energyprotein supplement optimizes the metabolic state of Romosinuano cows during the pre and postpartum period, and the productive performance, BW and BCS postpartum.

Key words: Romosinuano, nutrition, metabolic profile, prepartum, postpartum

\section{INTRODUCCIÓN}

La raza Romosinuano (Bos taurus) es una de las siete razas de bovinos criollos colombianos que se caracteriza por su aptitud cárnica, longevidad, temperamento dócil, y su potencial adaptativo a las zonas de vida del bosque seco tropical (bs-T) y húmedo tropical (bh-T) predominantes en el Caribe co- lombiano (Ossa et al., 2013), lo cual sugiere que su comportamiento productivo y reproductivo sea considerado como aceptable, debido a las contingencias que conllevan los ambientes tropicales (Martínez-Rocha et al., 2020).

La región Caribe colombiana contribuye con el $30 \%$ de la producción bovina a nivel nacional (ICA, 2020) y los sistemas pro- 
ductivos de cría con base en el uso de las razas criollas, como el Romosinuano, representan una importante participación, destacándose por su potencial productivo de carne con adecuada calidad organoléptica y sensorial; sin embargo estos sistemas se desarrollan bajo diversas condiciones agroecológicas de infraestructura donde el manejo de la alimentación, reproducción, y sanidad deben ser atendidos para optimizar la producción animal (Parra-Cortés et al., 2019).

Los cambios fisiológicos que ocurren en las vacas durante el pre y posparto incurren en alteraciones metabólicas condicionadas por desequilibrios homeostáticos, dados entre otros factores por desbalances entre los requerimientos y el consumo de nutrientes, que afectan la actividad reproductiva posparto (Moore y DeVries, 2020). En este aspecto, la incapacidad del animal en mantener la homeorresis y el desbalance nutricional puede evaluarse mediante el comportamiento de los indicadores metabólicos, tanto energéticos y proteicos y de función hepática (Puppel y Kuczyñska, 2016; Klein et al., 2021). De forma paralela, la medición de la condición corporal (CC) es un importante método de evaluación subjetiva que permite cuantificar las reservas corporales de energía endógena (Pfeifer et al., 2021).

Los desequilibrios homeostáticos que ocurren durante el último tercio de la gestación y en el posparto temprano, caracterizados principalmente por el balance energético negativo (BEN), una condición que representa cambios importantes en la concentración de metabolitos y en la disminución de la condición corporal (CC) (Clariget et al., 2020), estarían relacionados principalmente a condiciones de restricción alimentaria, que según Claramunt et al. (2020) pueden contrarrestarse mediante el adecuado manejo de la disponibilidad de forraje en la pradera y, complementariamente, con la oferta de alimentos energéticos y proteicos, que además de enriquecer la calidad nutricional de las dietas basadas en forrajes tropicales, contribu- yen a aumentar el consumo y la digestibilidad de los alimentos y el desempeño productivo en periodos críticos como la gestación y posparto en vacas (Mestra et al., 2013; Sotelo et al., 2018). Sin embargo, estudios relacionados con los efectos de la suplementación en vacas de aptitud cárnica, durante el pre y posparto en condiciones del trópico, presentan resultados controversiales sobre la reducción del BEN, y el desempeño productivo y reproductivo de los animales (Lopes et al., 2016).

Por lo anterior, el objetivo de este estudio fue evaluar la influencia del manejo alimenticio mediante la suplementación energético-proteica desde el preparto hasta el posparto sobre el desempeño productivo en términos de ganancia de peso, condición corporal, y el comportamiento del perfil metabólico de vacas Romosinuano en condiciones de pastoreo en el valle medio del río Sinú.

\section{Materiales y Métodos}

\section{Localización del Estudio}

El estudio se realizó en el Centro de Investigación Turipaná - AGROSAVIA, localizado en el departamento de Córdoba, en el Nor-occidente Colombia, subregión del valle medio del Sinú. La zona presenta una topografía plana, con altitud de $20 \mathrm{msnm}$, temperatura de $28{ }^{\circ} \mathrm{C}$, humedad relativa de $87 \%$ y precipitación pluvial anual de $1120 \mathrm{~mm}$. Todos los procedimientos experimentales de este estudio fueron aprobados por el Comité de Bioética de la Corporación Colombiana de Investigación Agropecuaria - AGROSAVIA.

\section{Animales, Alimentación y Tratamientos}

Se utilizaron 30 vacas multíparas Romosinuano del Banco de Germoplasma Bovino del Centro de Investigación Turipaná, de 3-5 años, con $250 \pm 23$ días de gestación y peso de $458 \pm 13 \mathrm{~kg}$. 
Cuadro1. Composición química ${ }^{1}$ de los alimentos (porcentaje de materia seca, \%MS) y nutrientes del suplemento ofrecido a vacas Romosinuano, según tratamiento experimental

\begin{tabular}{|c|c|c|c|c|c|c|c|}
\hline & $\begin{array}{l}\text { MS } \\
(\%)\end{array}$ & $\begin{array}{l}\mathrm{PC} \\
(\%)\end{array}$ & $\begin{array}{l}\mathrm{EE} \\
(\%)\end{array}$ & $\begin{array}{c}\text { FDN } \\
(\%)\end{array}$ & $\begin{array}{l}\text { FDA } \\
(\%)\end{array}$ & $\begin{array}{c}\mathrm{CNZ} \\
(\%)\end{array}$ & $\begin{array}{c}\text { EM } \\
\text { (Mcal/kg/MS) }\end{array}$ \\
\hline Angleton & 26.3 & 8.78 & 1.80 & 61.5 & 32.8 & 13.8 & 2.03 \\
\hline Salvado de arroz & 90.0 & 13.6 & 16.0 & 20.6 & 9.2 & 8.6 & 2.86 \\
\hline Torta de algodón & 90.3 & 47.0 & 3.62 & 33.7 & 11.7 & 6.4 & 2.04 \\
\hline \multirow[t]{3}{*}{ Melaza } & 73.0 & 3.0 & 1.0 & - & - & 10.1 & 3.28 \\
\hline & & \multicolumn{5}{|c|}{ Suplemento energético proteico ${ }^{2}$} & \\
\hline & & $\mathrm{TC}$ & & $\mathrm{T} 1$ & $\mathrm{~T} 2$ & & \\
\hline \multicolumn{8}{|c|}{ Ingredientes (kg de MS) } \\
\hline \multicolumn{2}{|c|}{ Salvado de arroz } & - & & 1.25 & 1.5 & & \\
\hline \multicolumn{2}{|c|}{ Torta de algodón } & - & & 1.1 & 1.1 & & \\
\hline \multicolumn{2}{|l|}{ Melaza } & - & & - & 0.2 & & \\
\hline \multicolumn{8}{|l|}{ Nutrientes $^{3}$} \\
\hline \multicolumn{2}{|c|}{ MS (kg/día) } & - & & 2.35 & 2.8 & & \\
\hline \multicolumn{2}{|l|}{ PC (kg/día) } & - & & 0.687 & 0.727 & & \\
\hline \multicolumn{2}{|c|}{ EM (Mcal/ración) } & - & & 5.92 & 7.18 & & \\
\hline
\end{tabular}

${ }^{1} \mathrm{MS}$ : materia seca; PC: proteína cruda, EE: extracto etéreo; FDN: fibra en detergente neutro; FDA: fibra en detergente ácido; Cnz: cenizas; EM: energía metabolizable (Mcal/kg/MS)

${ }^{2}$ Composición del suplemento energético-proteico, como ofrecido $(\mathrm{kg} / \mathrm{MS} / \mathrm{d})$ durante el pre y posparto

${ }^{3}$ Nutrientes totales diarios proporcionados en la ración por el suplemento alimenticio en animales del $\mathrm{T} 1$ y $\mathrm{T} 2$

Para el manejo alimenticio y suplementación, el tamaño muestral o número de animales representativos a usar en el ensayo se determinó mediante la siguiente ecuación descrita por Melo et al. $(2020): r=\left(2 Z^{2}(1-\alpha /\right.$ 2) $\left.\sigma^{2}\right) / \mathrm{d}^{2}$, donde $r$ : corresponde al número mínimo de replicas, $Z^{2}$ es el valor de una distribución normal estándar al nivel de confianza dado, $1-\alpha / 2$ es el valor conocido como nivel de confianza, $\sigma^{2}$ es la desviación estándar de la variable de interés y $\mathrm{d}^{2}$ es el nivel de precisión deseado en la comparación.

Los animales fueron asignados aleatoriamente a uno de los tres tratamientos nutricionales de la siguiente manera: TC, grupo control, pastoreo sin suplementación; T1, pastoreo+ suplementación energéticoproteica $(2.35 \mathrm{~kg} /$ animal $/$ día $)$; T2, pastoreo + suplementación energético-proteica $(2.8 \mathrm{~kg} /$ animal/día) (Cuadro 1). Las cantidades de suplemento suministrado en las dietas del T1 y T2 se formularon con diferencias de1 5.8\% en la oferta de proteína cruda $(\mathrm{kg})$ y del $21 \%$ de energía metabolizable (Mcal), a fin de evaluar el efecto de los nutrientes disponibles en cada dieta sobre las variables productivas de animales durante el pre y posparto. Cada grupo estuvo conformado por 10 vacas y el periodo experimental comprendió desde el día 30 del preparto hasta el día 60 posparto de vacas, junto con sus crías en amamantamiento ad libitum. 
El suplemento consistió en una mezcla de subproductos agroindustriales como salvado de arroz (Oryza sativa), torta de algodón (Gossypum sp) y melaza de caña (Sacharum oficiarum), balanceado que suplía los requerimientos nutricionales de acuerdo con el NRC (2000). Los animales estuvieron en pastoreo rotacional intensivo de pasto Angleton (Dichanthium aristatum) en un potrero de $18.75 \mathrm{ha}$. Cada tratamiento tuvo un área de 6.25 ha con 5 subdivisiones de 5 días de ocupación y 20 de descanso. El suplemento se ofreció diariamente a los animales del T1 y T2 durante el horario de 07:00 a 08:30 h en comederos móviles grupales con espacio suficiente para evitar competencia o dominancia entre animales, y el consumo de suplemento se registró a las 10:00 h. La disponibilidad del forraje $(\mathrm{kg} / \mathrm{MS} / \mathrm{ha})$ se evaluó en los potreros asignados, mediante la metodología de disponibilidad por frecuencia propuesta por Franco et al. (2006). Además, el consumo de la materia seca (MS) del forraje fue estimado por el método agronómico (Hammeleers, 2002).

\section{Calidad Nutricional del Forraje y Suple- mento}

Se colectaron muestras representativas del forraje simulando el pastoreo (hand plucking) de cada potrero cada 30 días para evaluar la calidad nutricional. Las muestras fueron secadas en estufa de ventilación forzada (Binder FD 240) a $60{ }^{\circ} \mathrm{C}$ por $48 \mathrm{~h}$, molidas a $1 \mathrm{~mm}$ y enviadas al laboratorio de Química Analítica del Centro de Investigación Turipaná. Los análisis químicos en estas muestras, así como en las de los componentes del concentrado se hicieron según los métodos de referencia de la AOAC (2005).

\section{Consumo y Digestibilidad de Nutrientes}

Los resultados del análisis químico del forraje y de los suplementos se registraron en el software Cornell Net Carbohydrate and Protein System (CNCPS v. 6.5.5.1 de la Universidad de Cornell, EEUU (Fox et al., 2004), para predecir el consumo, digestibilidad y metabolismo de los nutrientes.

\section{Parámetros Productivos}

La evaluación del peso corporal (Pcorp) y condición corporal (CC) de los animales se realizó el día 30 del preparto y 60 del posparto. El Pcorp se registró utilizando báscula con capacidad de $2000 \mathrm{~kg}$, y la CC fue evaluada por una misma persona mediante una escala de calificación de 1 a 9 (Richards et al., 1986).

\section{Perfil Metabólico}

Se determinaron las concentraciones de los componentes del metabolismo energético (glucosa [GLU], colesterol [COL], betahidroxibutirato [BHB), ácidos grasos no esterificados [NEFAs]), proteico (proteína total [PT], urea) y enzimático (aspartato aminotransferasa [AST] y alanina aminotransferasa [ALT]). A los animales en ayuno se les tomaron muestras de sangre (10 $\mathrm{ml})$ de la vena coccígea utilizando tubos al vacío sin anticoagulante (Vacutainer $\left.{ }^{\circledR}\right)$ los días 30 preparto y $5,12,27,42$ y 60 posparto. Las muestras fueron colectadas entre las 08:00 y 11:00 h. El suero fue separado mediante centrifugación a $15000 \mathrm{~g}$ durante 15 min (LC-04R Scientific ${ }^{\circledR}$ ) y se almacenaron por duplicado en tubos eppendorf $(2 \mathrm{ml})$ a $20^{\circ} \mathrm{C}$. El análisis se hizo mediante reactivos comerciales y espectrofotometría, a través de analizador químico automático Rayto ${ }^{\circledR}$ (Shenzhen, China). Mediante técnicas enzimáticas colorimétricas se determinaron las concentraciones séricas de: proteína (TP245), glucosa (Gluc-PAP GL2623), betahidroxibutirato (Ranbut RB1007), NEFAs (FA115), colesterol (CH203), urea (UR446), AST (AS2359) y ALT (AL2360). Todos los reactivos analíticos fueron del laboratorio Randox ${ }^{\circledR}$ (Antrim, UK). 


\section{Análisis Estadístico}

Los datos se analizaron mediante un análisis de varianza (PROC GLM) de medidas repetidas para determinar las variaciones en los indicadores productivos y metabólicos de los animales a través del tiempo y el efecto de los tratamientos sobre las variables de interés. Se realizaron pruebas de normalidad (Shapiro-Wilk) y homocedasticidad (Levene). Los datos se analizaron por tratamiento (TC T1 y T2) y durante los periodos de preparto y posparto. Se compararon las medias de los indicadores metabólicos séricos durante los dos periodos mediante el test de Tukey y Kramer en el programa Infostat ${ }^{\circledR}$ con un nivel de significancia de $5 \%$.

\section{Resultados y Discusión}

\section{Consumo y Digestibilidad de Nutrientes}

Durante el periodo experimental, el pasto Angleton (Dichanthium aristatum) presentó una disponibilidad de $61.5 \%$ de FDN, que se indica dentro de los valores aceptables para gramíneas tropicales, así como 2.03 Mcal $/ \mathrm{kg} / \mathrm{MS}$ de EM, y $8.78 \%$ de PC (Cuadro 1), lo cual representó un aporte de $87.8 \mathrm{~g}$ de $\mathrm{PC} / / \mathrm{kg} \mathrm{MS}$, levemente superior al rango mínimo 6-8\% de MS para mantener el crecimiento microbiano y promover la digestión de fibras carbohidratos del forraje (Detmann et al., 2014).

El aporte diario de nutrientes del suplemento para animales del T1 y T2 se presentan en el Cuadro 1. La masa de forraje disponible para cada tratamiento se muestra en el Cuadro 2, la cual permitió consumir una ración de pasto de $11.1,10.5$ y $10.3 \mathrm{~kg} / \mathrm{ani}$ $\mathrm{mal} /$ día para TC, T1 y T2 (Cuadro 2).

\section{Parámetros Productivos}

No hubo diferencias significativas entre tratamientos para peso $(\mathrm{p}=0.4557)$ y condición corporal $(\mathrm{p}=0.1893)$. Sin embargo, en el día 60 posparto las vacas de T1 y T2 presentaron $30.6 \mathrm{~kg}$ más de Pcorp en promedio que las vacas de $\mathrm{TC}(\mathrm{p}=0.0343)$ y un punto más en $\mathrm{CC}(\mathrm{p}=0.0292)$ (Cuadro 3).

El menor Pcorp y CC de animales del TC en el posparto concuerda con lo indicado por Carizi et al. (2019), quienes señalan una pérdida progresiva de peso y CC luego del parto, lo cual se debería principalmente por el consumo restringido de NDT, dado que la alimentación estuvo basada únicamente en el forraje de la pradera, con aportes deficitarios de nutrientes para cubrir los requerimientos de energía (consumo de 6249 g/día de NDT $56.3 \%$ del consumo de MS/d) (Cuadro 2), inferior a lo recomendado durante el posparto por el NRC (2000) del 60-65\%, lo que limitó el desempeño de los animales en la ganancia de peso.

Respecto a la disminución de la CC en animales del TC, Diskin y Kenny (2016) indican que el amamantamiento es una condición que afecta negativamente la $\mathrm{CC}$ de las vacas cuando no tienen un adecuado manejo nutricional. Asimismo, los resultados del presente estudio concuerdan con Sotelo et al. (2018), quienes encontraron disminución de la $\mathrm{CC}$ al posparto en vacas de carne con ternero al pie y alimentadas solo con pasto. Las vacas de $\mathrm{T} 1$ y $\mathrm{T} 2$ se beneficiaron con una mayor disponibilidad de nutrientes diarios (Cuadro 2), generando un mayor efecto anabólico en el metabolismo de los animales, logrando un aumento en el Pcorp y CC de animales del T1 y T2 al final del estudio (día 60 postparto), tal y como lo reportan Oliveira et al. (2010) en vacas Canchim suplementadas durante el pre y posparto en condiciones de pastoreo. 
Cuadro 2. Consumo y digestibilidad de nutrientes de vacas Romosinuano al pastoreo y suplementadas con balanceado durante el pre y posparto (Córdoba, Colombia)

\begin{tabular}{lccc}
\hline & $\mathrm{TC}$ & $\mathrm{T} 1$ & $\mathrm{~T} 2$ \\
\hline Forraje disponible (kg/MS/ha) & 555.2 & 524.8 & 516.15 \\
Consumo de nutrientes ${ }^{1}$ & & & \\
$\quad$ Materia seca del forraje $(\mathrm{kg} / \mathrm{d})$ & 11.1 & 10.5 & 10.3 \\
$\quad$ Materia seca del suplemento $(\mathrm{kg} / \mathrm{d})$ & - & 2.35 & 2.8 \\
$\quad$ Materia seca total (MST) & 11.1 & 12.85 & 13.1 \\
$\quad$ Proteína cruda (g/d) & 900 & 1608 & 1631 \\
$\quad$ Extracto etéreo (g/d) & 150 & 372 & 415 \\
Carbohidratos no fibrosos (g/d) & 1820 & 2160 & 2450 \\
Composición ${ }^{2}$ & & & \\
EM (Mcal/d) & 23.4 & 29.5 & 31.1 \\
ENm (Mcal/d) & 13.2 & 18.3 & 19.1 \\
PM (g/d) & 837 & 1220 & 1283 \\
NDT (g/d) & 6249 & 7914 & 8262 \\
NDT (\%) & 56.3 & 61.0 & 63.0 \\
\hline
\end{tabular}

TC, grupo control, pastoreo sin suplementación; T1, pastoreo+ suplementación energético proteica

( $2.35 \mathrm{~kg} /$ animal/día); T2, pastoreo + suplementación energético proteica ( $2.8 \mathrm{~kg} /$ animal/día)

${ }^{1}$ Consumo de nutrientes analizado mediante software CNCPS con base al análisis de los alimentos

2 EM: energía metabolizable (Mcal/d); ENm: energía neta de mantenimiento y lactancia (Mcal/d); PM: proteína metabolizable; NDT: nutrientes digestibles totales

Cuadro 3. Peso y condición corporal (CC) de vacas Romosinuano al pastoreo y suplementadas con balanceado durante el pre y posparto (Córdoba, Colombia)

\begin{tabular}{ccccc}
\hline \multirow{2}{*}{ Tratamientos } & \multicolumn{2}{c}{ Preparto $^{1}$} & \multicolumn{2}{c}{$\begin{array}{c}\text { Posparto }^{2} \\
\text { (30 días previos) }\end{array}$} \\
\cline { 2 - 5 } & Peso $(\mathrm{kg})$ & CC & Peso $(\mathrm{kg})$ & CC \\
\hline TC & $467.4^{\mathrm{a}}$ & $6.5^{\mathrm{a}}$ & $440.8^{\mathrm{a}}$ & $5.5^{\mathrm{a}}$ \\
$\mathrm{T} 1$ & $455.6^{\mathrm{b}}$ & $6.0^{\mathrm{a}}$ & $468.5^{\mathrm{b}}$ & $7.0^{\mathrm{b}}$ \\
$\mathrm{T} 2$ & $451.8^{\mathrm{b}}$ & $6.0^{\mathrm{a}}$ & $474.4^{\mathrm{b}}$ & $7.0^{\mathrm{b}}$ \\
\hline Valor $p$ & 0.4557 & 0.1893 & 0.0343 & 0.0292 \\
\hline
\end{tabular}

TC, grupo control, pastoreo sin suplementación; T1, pastoreo+ suplementación energético proteica ( $2.35 \mathrm{~kg} /$ animal/día); T2, pastoreo + suplementación energético proteica ( $2.8 \mathrm{~kg} /$ animal/día)

$a, b$ Medias con letra diferente dentro de la fila difieren estadísticamente $(p<0.05)$ 


\section{Perfil Metabólico}

Los valores medios \pm error estándar de las concentraciones séricas de los componentes del metabolismo energético, proteico y enzimático en el pre y posparto se presentan en el Cuadro 4.

\section{Glucosa (GLU)}

El valor de glucosa fue más elevado en el preparto en las vacas de T2 (Cuadro 4). Asimismo, este valor fue superior al reportado por García et al. (2019) en vacas cebú y Simmental $\mathrm{x}$ cebú en pastoreo durante el último tercio de gestación $(2.42 \pm 0.13 \mathrm{mmol} / \mathrm{l}) \mathrm{y}$ por Piao et al. (2015) en vacas de aptitud cárnica Hanwoo al final de la gestación $(2.5 \pm 0.01 \mathrm{mmol} / \mathrm{l})$.

Los valores se mantuvieron estables y sin diferencia estadística durante el posparto (Cuadro 4), similares a los valores reportados por Samadi et al. (2013) de $3.4 \pm 0.03 \mathrm{mmol} / 1$ en vacas de aptitud cárnica, pero ligeramente superiores a las reportadas por Quintela et al. (2011) en vacas Rubia Gallega.

\section{Colesterol}

Al preparto, el grupo de animales del T2 presentó los mayores niveles séricos de COL ( $\mathrm{p}=0.0036$ ) (Cuadro 4), similares a los valores reportados por Piao et al., (2015) en vacas Hanwoo $(3.39 \pm 0.93 \mathrm{mmol} / \mathrm{l})$ y por Garcia et al. (2019) $(3.4 \pm 0.23 \mathrm{mmol} / \mathrm{l})$ en vacas cruzadas cebú x Simmental al preparto.

En el postparto, las mayores concentraciones de COL se encontraron el día 5 en animales del T2 $(\mathrm{p}=0.001)$, con valores mayores a los reportados por Moura et al. (2020) en vacas de carne suplementadas al posparto $(3.32 \pm 0.26 \mathrm{mmol} / \mathrm{l})$. Asimismo, el COL en animales del T1 se encontró incrementado al día 60 posparto (Cuadro 4), con valores similares a los reportados por Mapfumo et al. (2017) (4.8 $80.33 \mathrm{mmol} / \mathrm{l})$ en vacas Boran (Bos taurus africanus).
El incremento de las concentraciones de COL durante el posparto es un tema muy discutido, en razón a que algunos autores relacionan esta condición con el mejor equilibrio energético (Silveira et al., 2012), mientras que otros indican que este efecto estaría relacionado con deficiencias en el consumo de energía con pérdidas de la condición corporal, como un mecanismo de movilización de reservas corporales para atender las demandas de nutrientes durante la lactancia (Berman, 2011; Klein et al., 2021). En este estudio, el incremento de COL del T1 al día 60 posparto podría asociarse a una mayor demanda por consumo de energía que la suministrada en la dieta, lo cual se evidenció en el menor consumo de grasa o extracto etéreo de $-371.6 \mathrm{~g} / \mathrm{d}$ al compararse con el consumo de los animales del T2 (Cuadro 4) y no con un BEN, debido a que la CC de los animales del T1 se incrementó durante el posparto (Cuadro 2).

\section{$\beta$-hidroxibutirato (BHB)}

Las concentraciones séricas de BHB en el preparto no difirieron entre tratamientos $(p=0.2537)$, presentando un valor promedio de $0.64 \pm 0.07$ (Cuadro 4), similar al reportado por Cervantes et al. (2014) en vacas mestizas $(0.69 \pm 0.09 \mathrm{mmol} / \mathrm{l})$. Las concentraciones séricas de BHB en el posparto fueron igualmente similares entre tratamientos $(\mathrm{p}=0.3503)$, aunque con mayores valores los días 5 y 12 posparto en el TC $(\mathrm{p}=0.0365)$. Este aumento en el TC obedece a la mayor movilización de tejido adiposo en respuesta al BEN que se evidenció por la restricción en el consumo de energía y NDT diarios, que correspondieron a $6249 \mathrm{~g}$ /día (Cuadro 2) con un déficit de -411g/día, para suplir el requerimiento mínimo del $60 \%$ de NDT y de energía neta $\mathrm{ENm}$ (Mcal/día) que correspondió a 13.2 Mcal. Este valor es inferior al consumo recomendado de $16 \mathrm{Mcal}$ de ENm diaria para cubrir los requerimientos del mantenimiento y lactancia (NRC, 2000). 
Cuadro 4. Concentraciones séricas de componentes del metabolismo energético, proteico y enzimático en vacas Romosinuano suplementadas con balanceado durante el pre y posparto (Córdoba, Colombia)

\begin{tabular}{ccccccc}
\hline \multicolumn{3}{c}{ Glucosa (mmol/l) } & \multicolumn{3}{c}{ Colesterol (mmol/l) } \\
\hline Día $^{1}$ & TC & T1 & T2 & TC & T1 & T2 \\
\hline-30 & $2.75 \pm 0.316^{\mathrm{a}}$ & $3.45 \pm 0.36^{\mathrm{a}}$ & $4.65 \pm 0.33^{\mathrm{b}}$ & $2.31 \pm 0.18^{\mathrm{a}}$ & $1.95 \pm 0.216^{\mathrm{a}}$ & $3.06 \pm 0.20^{\mathrm{b}}$ \\
5 & $2.97 \pm 0.46^{\mathrm{a}}$ & $3.18 \pm 0.46^{\mathrm{a}}$ & $2.70 \pm 0.46^{\mathrm{a}}$ & $2.15 \pm 0.34^{\mathrm{ab}}$ & $2.66 \pm 0.34^{\mathrm{ab}}$ & $3.72 \pm 0.34^{\mathrm{c}}$ \\
12 & $3.02 \pm 0.46^{\mathrm{a}}$ & $2.50 \pm 0.46^{\mathrm{a}}$ & $3.43 \pm 0.46^{\mathrm{a}}$ & $2.41 \pm 0.34^{\mathrm{ab}}$ & $2.82 \pm 0.34^{\mathrm{ab}}$ & $2.89 \pm 0.34^{\mathrm{ab}}$ \\
27 & $3.58 \pm 0.43^{\mathrm{a}}$ & $3.14 \pm 0.46^{\mathrm{a}}$ & $3.49 \pm 0.46^{\mathrm{a}}$ & $2.67 \pm 0.32^{\mathrm{ab}}$ & $3.12 \pm 0.34^{\mathrm{b}}$ & $3.43 \pm 0.34^{\mathrm{b}}$ \\
42 & $2.35 \pm 0.43^{\mathrm{a}}$ & $2.10 \pm 0.43^{\mathrm{a}}$ & $3.00 \pm 0.46^{\mathrm{a}}$ & $2.94 \pm 0.34^{\mathrm{ab}}$ & $3.11 \pm 0.32^{\mathrm{b}}$ & $2.96 \pm 0.34^{\mathrm{ab}}$ \\
60 & $2.44 \pm 0.46^{\mathrm{a}}$ & $3.01 \pm 0.46^{\mathrm{a}}$ & $3.25 \pm 0.40^{\mathrm{a}}$ & $3.97 \pm 0.34^{\mathrm{bc}}$ & $4.80 \pm 0.34^{\mathrm{d}}$ & $3.52 \pm 0.30^{\mathrm{bc}}$
\end{tabular}

\begin{tabular}{ccccccc}
\hline & \multicolumn{3}{c}{ BHB (mmol/1) } & \multicolumn{3}{c}{ NEFAs (mmol/l) } \\
\hline Día & TC & T1 & T2 & TC & T1 & T2 \\
\hline-30 & $0.58 \pm 0.07^{\mathrm{a}}$ & $0.61 \pm 0.08^{\mathrm{a}}$ & $0.75 \pm 0.07^{\mathrm{a}}$ & $0.40 \pm 0.05^{\mathrm{ab}}$ & $0.45 \pm 0.06^{\mathrm{ab}}$ & $0.32 \pm 0.05^{\mathrm{a}}$ \\
5 & $0.82 \pm 0.08^{\mathrm{b}}$ & $0.56 \pm 0.08^{\mathrm{a}}$ & $0.60 \pm 0.08^{\mathrm{a}}$ & $0.39 \pm 0.06^{\mathrm{a}}$ & $0.35 \pm 0.06^{\mathrm{a}}$ & $0.36 \pm 0.06^{\mathrm{a}}$ \\
12 & $0.91 \pm 0.08^{\mathrm{c}}$ & $0.73 \pm 0.08^{\mathrm{ab}}$ & $0.56 \pm 0.08^{\mathrm{a}}$ & $0.41 \pm 0.06^{\mathrm{ab}}$ & $0.26 \pm 0.06^{\mathrm{a}}$ & $0.44 \pm 0.06^{\mathrm{ab}}$ \\
27 & $0.75 \pm 0.08^{\mathrm{b}}$ & $0.74 \pm 0.08^{\mathrm{b}}$ & $0.78 \pm 0.08^{\mathrm{b}}$ & $0.40 \pm 0.06^{\mathrm{ab}}$ & $0.35 \pm 0.06^{\mathrm{a}}$ & $0.36 \pm 0.06^{\mathrm{a}}$ \\
42 & $0.66 \pm 0.08^{\mathrm{ab}}$ & $0.81 \pm 0.08^{\mathrm{c}}$ & $0.65 \pm 0.08^{\mathrm{ab}}$ & $0.33 \pm 0.06^{\mathrm{a}}$ & $0.43 \pm 0.06^{\mathrm{ab}}$ & $0.34 \pm 0.06^{\mathrm{a}}$ \\
60 & $0.55 \pm 0.08^{\mathrm{a}}$ & $0.70 \pm 0.08^{\mathrm{ab}}$ & $0.63 \pm 0.08^{\mathrm{ab}}$ & $0.38 \pm 0.06^{\mathrm{a}}$ & $0.51 \pm 0.06^{\mathrm{bc}}$ & $0.45 \pm 0.06^{\mathrm{ab}}$
\end{tabular}

\begin{tabular}{|c|c|c|c|c|c|c|}
\hline \multicolumn{4}{|c|}{ Proteína total $(\mathrm{g} / \mathrm{dl})$} & \multicolumn{3}{|c|}{ Urea (mmol/l) } \\
\hline Día & $\mathrm{TC}$ & $\mathrm{T} 1$ & $\mathrm{~T} 2$ & TC & $\mathrm{T} 1$ & $\mathrm{~T} 2$ \\
\hline-30 & $7.78 \pm 0.23^{\mathrm{a}}$ & $7.47 \pm 0.27^{\mathrm{a}}$ & $7.56 \pm 0.25^{\mathrm{a}}$ & $4.98 \pm 0.34^{b}$ & $3.61 \pm 0.39^{\mathrm{a}}$ & $3.93 \pm 0.36^{\mathrm{a}}$ \\
\hline 5 & $8.05 \pm 0.31^{\mathrm{b}}$ & $8.44 \pm 0.31^{b}$ & $8.83 \pm 0.31^{\mathrm{bc}}$ & $3.68 \pm 0.44^{\mathrm{a}}$ & $3.99 \pm 0.44^{\mathrm{a}}$ & $3.94 \pm 0.44^{\mathrm{a}}$ \\
\hline 12 & $8.09 \pm 0.31^{\mathrm{b}}$ & $8.21 \pm 0.31^{\mathrm{b}}$ & $9.03 \pm 0.31^{\mathrm{c}}$ & $2.98 \pm 0.44^{\mathrm{a}}$ & $4.20 \pm 0.44^{\mathrm{ab}}$ & $3.39 \pm 0.44^{\mathrm{a}}$ \\
\hline 27 & $8.02 \pm 0.29^{b}$ & $7.90 \pm 0.31^{\mathrm{a}}$ & $7.91 \pm 0.31^{\mathrm{a}}$ & $4.00 \pm 0.42^{\mathrm{ab}}$ & $3.91 \pm 0.44^{\mathrm{a}}$ & $3.95 \pm 0.44^{\mathrm{a}}$ \\
\hline 42 & $8.54 \pm 0.3^{\mathrm{b}}$ & $8.23 \pm 0.29^{b}$ & $7.90 \pm 0.31^{\mathrm{a}}$ & $4.54 \pm 0.44^{\mathrm{c}}$ & $3.63 \pm 0.42^{\mathrm{a}}$ & $3.83 \pm 0.44^{\mathrm{a}}$ \\
\hline \multirow[t]{2}{*}{60} & $7.80 \pm 0.31^{\mathrm{a}}$ & $7.67 \pm 0.31^{\mathrm{a}}$ & $7.93 \pm 0.37^{\mathrm{a}}$ & $4.38 \pm 0.44^{\mathrm{b}}$ & $3.92 \pm 0.44^{\mathrm{a}}$ & $3.72 \pm 0.39^{\mathrm{a}}$ \\
\hline & \multicolumn{3}{|c|}{ AST (U/L) } & \multicolumn{3}{|c|}{$\operatorname{ALT}(\mathrm{U} / \mathrm{L})$} \\
\hline Día & $\mathrm{TC}$ & T1 & $\mathrm{T} 2$ & TC & $\mathrm{T} 1$ & $\mathrm{~T} 2$ \\
\hline-30 & $67.10 \pm 4.77^{\mathrm{a}}$ & $68.09 \pm 5.51^{\mathrm{ab}}$ & $63.35 \pm 5.10^{\mathrm{ab}}$ & $17.47 \pm 1.50^{\mathrm{a}}$ & $21.63 \pm 1.74^{\mathrm{a}}$ & $19.85 \pm 1.61^{\mathrm{a}}$ \\
\hline 5 & $76.31 \pm 7.46^{\mathrm{ab}}$ & $77.09 \pm 7.46^{\mathrm{ab}}$ & $99.27 \pm 7.46^{\mathrm{c}}$ & $20.89 \pm 1.81^{\mathrm{a}}$ & $24.86 \pm 1.81^{\mathrm{ab}}$ & $21.97 \pm 1.8^{\mathrm{a}}$ \\
\hline 12 & $78.12 \pm 27.46^{\mathrm{ab}}$ & $74.95 \pm 7.46^{\mathrm{ab}}$ & $64.36 \pm 7.46^{\mathrm{ab}}$ & $20.43 \pm 1.81^{\mathrm{a}}$ & $22.64 \pm 1.81^{\mathrm{a}}$ & $22.01 \pm 1.81^{\mathrm{a}}$ \\
\hline 27 & $82.83 \pm 6.98^{\mathrm{bc}}$ & $79.19 \pm 7.46^{\mathrm{abc}}$ & $78.20 \pm 7.46^{\mathrm{ab}}$ & $22.98 \pm 1.70^{\mathrm{a}}$ & $23.59 \pm 1.81^{\mathrm{a}}$ & $22.01 \pm 1.81^{\mathrm{a}}$ \\
\hline 42 & $82.32 \pm 7.4^{\mathrm{bc}}$ & $82.50 \pm 6.98^{\mathrm{bc}}$ & $79.31 \pm 7.4^{\mathrm{abc}}$ & $26.56 \pm 1.81^{\mathrm{bcd}}$ & $24.63 \pm 1.7^{\mathrm{bc}}$ & $24.65 \pm 1.81^{\mathrm{bc}}$ \\
\hline 60 & $75.09 \pm 7.46^{\mathrm{ab}}$ & $75.70 \pm 7.46^{\mathrm{abc}}$ & $82.45 \pm 6.48^{\mathrm{bc}}$ & $25.65 \pm 1.81^{\mathrm{bc}}$ & $22.18 \pm 1.81^{\mathrm{a}}$ & $27.32 \pm 1.60^{\mathrm{d}}$ \\
\hline
\end{tabular}

1 Días pre y posparto

$a, b, c$ Medias con letra diferente dentro de filas y metabolito difieren estadísticamente $(p<0.05)$

Media general \pm error estándar de la media

Betahidroxibutirato [BHB), ácidos grasos no esterificados [NEFAS]), aspartato aminotransferasa [AST], alanina aminotransferasa [ALT] 
En los animales del T1 se encontró un leve incremento en las concentraciones de BHB en el día 42 , pero inferior al $\leq 1.4 \mathrm{mmol} / 1$ reportado por McArt et al. (2013) como indicador de incidencia de enfermedades metabólicas en rebaños al posparto. En este sentido, este incremento estaría relacionado con las condiciones propias de demandas de energía durante el periodo de la lactancia (Gross et al., 2011) y no con un BEN, debido a que los animales suplieron sus requerimientos de NDT (g/día) y de ENm (Mcal/kg/MS) y aumentaron la ganancia de peso en $12.9 \mathrm{~kg}$ y un punto en la CC (Cuadro 3).

\section{Ácidos grasos no esterificados (NEFAs)}

Los valores de NEFAs fueron similares entre tratamientos en el preparto $(\mathrm{p}=0.2283)$ y con un promedio de $0.39 \pm 0.05$ (Cuadro 4). Asimismo, estas concentraciones fueron inferiores a las descritas por Quintela et al. (2011) en vacas Rubia Gallega $(0.454 \pm 0.45$ $\mathrm{mmol} / \mathrm{l}$ ), y por Clariget et al. (2020) en vacas Hereford y Aberdeen Angus suplementadas desde el día 52 del preparto $(1.05 \pm$ $0.05 \mathrm{mmol} / \mathrm{l})$. Tampoco se encontraron diferencias significativas entre tratamientos $(\mathrm{p}=0.9071)$, con un valor promedio de $0.38 \pm 0.02$. No obstante, se observó un leve incremento en $\mathrm{T} 1$ al día 60 posparto, que coincidió con el valor de NEFAs reportado por Lopes et al. (2016) en vacas Nellore con suplementación proteica (320 g de PC/animal/día).

Los NEFAs corresponden a uno de los metabolitos comúnmente referenciados para evaluar la presentación de BEN en vacas, condición que es muy frecuente durante el preparto y en la lactancia de vacas lecheras de alta producción, cuando no pueden suplir sus requerimientos nutricionales para la producción (Waterman y Butler, 2010), situación que se contrasta en vacas de aptitud cárnica, debido a las menores demandas de energía. Durante las fases del pre y posparto del presente estudio, las concentraciones de NEFAs fueron inferiores a $0.40 \mathrm{mmol} / 1$, coincidiendo con valores reportados en otros estudios (Moura et al., 2020; Clariget et al., 2020; De
Lana et al., 2020) en vacas de aptitud cárnica en condiciones de trópico, concluyendo que valores $\leq 0.40 \mathrm{mmol} / 1$ no sugieren problemas relacionados con déficit energético en los animales.

\section{Proteina total (PT)}

Las concentraciones séricas de PT en el preparto no presentaron diferencias significativas entre tratamientos ( $\mathrm{p}=0.6815$ ) (Cuadro 4), con un valor promedio de 7.60 \pm 0.75 $\mathrm{g} / \mathrm{dl}$. Estos valores fueron similares a los reportados por Piao et al. (2015) en vacas durante el último tercio de gestación $(7.69 \pm 0.79$ $\mathrm{g} / \mathrm{dl}$ ), pero superiores a los indicados por García et al. (2019) en vacas de aptitud cárnica $(6.28 \pm 0.15 \mathrm{~g} / \mathrm{dl})$ durante el último tercio de gestación en condiciones de pastoreo extensivo y sin suplementación alimenticia.

Al día 5 del postparto se observó un incremento significativo en las concentraciones séricas de PT en T2 $(\mathrm{p}=0.0163)$ lo cual estaría asociado a la mayor ingesta de proteína de la dieta y por la mayor disponibilidad de proteína metabolizable o verdadera digerida pos-ruminalmente, que correspondió a 1283 g/día de PM (Cuadro 2). Por otro lado, las concentraciones séricas de PT en general durante el posparto fueron superiores a las reportadas por Mapfumo et al. (2017) en vacas Boran $(7.11 \pm 0.11 \mathrm{mmol} / \mathrm{l})$ y por Moura et al. (2020) en vacas Nellore $(6.74 \pm 0.13$ $\mathrm{mmol} / \mathrm{l})$.

\section{Urea}

La concentración sérica de urea en el preparto fue mayor en los animales del TC $(\mathrm{p}=0.0334)$ (Cuadro 4) y similar al valor reportado por Quintela et al. (2011) (4.65 1.73 $\mathrm{mmol} / \mathrm{l}$ ) en vacas Rubia Gallega en pastoreo y sin suplementación. Asimismo, las concentraciones de urea en animales de los T1 y T2 fueron similares al rango reportado por Mapfumo et al. (2017) en vacas Nguni $(3.9 \pm 0.26 \mathrm{mmol} / \mathrm{l})$. La concentración de urea fue igualmente mayor en animales del TC al día 42 ( $p=0.0442)$. Según Garcia y Bacallao 
(2010), el incremento de urea en animales del TC estaría relacionado con un desbalance energético-proteico de la dieta o por raciones deficitarias de energía o proteína, situación que frecuentemente se observa en rebaños alimentados con pastos tropicales.

Las vacas del TC presentaron la menor disponibilidad de PM $(837 \mathrm{~g} /$ día en promedio (Cuadro 2), siendo levemente inferior al límite mínimo requerido al postparto en vacas de aptitud cárnica ( 840 g/día; NRC, 2000). Además, estos animales no alcanzaron los requerimientos mínimos de $60 \%$ de NDT y 16.0 $\mathrm{Mcal} /$ día de ENm, y presentaron una pérdida de peso y de condición corporal durante el posparto (Cuadro 3), lo que reafirma un estado de desbalance energético en el TC al posparto.

\section{Aspartato aminotransferasa (AST)}

No hubo diferencias significativas entre tratamientos en el preparto $(\mathrm{p}=0.677)$ para las concentraciones enzimáticas de AST (Cuadro 4), siendo los valores similares a los valores de $67.81 \pm 17.08 \mathrm{U} / 1$ reportados por Quintela et al. (2011) y de 66.3 $\pm 3.16 \mathrm{U} / 1 \mathrm{de}$ Mapfumo et al. (2017). No obstante, en el día 5 posparto hubo un incremento de AST en animales del T2 ( $\mathrm{p}=0.0202)$, así como una tendencia a un incremento paulatino hasta el día 60 postparto en todos los tratamientos. Esto, según Wittwer (2000), no tendría importancia fisiológica debido a que no superaron los valores referenciales de $>120 \mathrm{U} / 1$. Por otro lado, los valores de AST al posparto fueron inferiores a los valores de $95.31 \pm 34.16$ $\mathrm{U} / 1$ reportados por Quintela et al. (2011) y de $74.0 \pm 18.0 \mathrm{U} / 1$ de Piao et al. (2015).

\section{Alanino aminotransferasa (ALT)}

Las concentraciones enzimáticas de ALT fueron similares entre tratamientos en el preparto $(\mathrm{p}=0.2129)$ (Cuadro 4), siendo los valores del TC similares a los reportados por Quintela et al. (2011) en vacas de aptitud cárnica sin suplementación. Por otro lado, los animales del $\mathrm{T} 1$ y T2 presentaron valores si- milares al valor de $22.85 \pm 3.7 \mathrm{U} / 1$ reportado por Mapfumo et al. (2017). Al posparto, las concentraciones de ALT fueron similares entre tratamientos ( $\mathrm{p}=0.6448)$; sin embargo, los animales del $\mathrm{T} 2$ al día 60 posparto presentaron valores mayores $(p=0.0018)$, los cuales fueron inferiores al valor de $44.9 \pm$ $1.64 \mathrm{U} / 1$ reportado por Mapfumo et al. (2017) en vacas Nguni.

El incremento de estas enzimas se relaciona a enfermedades de origen viral, o por situaciones de estrés (Mapfumo et al., 2017). Esto indica, que, bajo las condiciones experimentales del estudio, las vacas de TC, T1 y $\mathrm{T} 2$ no presentaron problemas sanitarios asociados con la condición fisiológica propias de las etapas de gestación y lactancia.

\section{Conclusiones}

- El presente estudio entrega por primera vez información sobre el perfil metabólico de vacas Romosinuano en etapas de pre y postparto, y su relación con la suplementación en condiciones del Caribe húmedo colombiano.

- Las vacas Romosinuano bajo regímenes de pastoreo y sin suplementación energético-proteica (TC) presentaron déficit en el consumo de NDT (-411g/día y ENm $-2.9 \mathrm{Mcal} /$ día) para atender las exigencias nutricionales y el mantenimiento de la homeostasis, con incrementos de metabolitos asociados con balance energético negativo, $\mathrm{BHB}$, urea, y disminución del peso corporal $(-26.6 \mathrm{~kg})$ y de la condición corporal en el posparto.

- El suministro de hasta $2.8 \mathrm{~kg} / \mathrm{d}$ de suplemento energético-proteico con densidades de 31.06 Mcal y $1283 \mathrm{~g}$ de proteína metabolizable diaria optimiza la ingesta de nutrientes del forraje, el desempeño productivo, ganancia de peso, condición corporal y el estatus metabólico durante el pre y posparto en las vacas Romosinuano mantenidas bajo las condiciones del valle medio del río Sinú. 


\section{Agradecimientos}

Los autores agradecen a la Corporación Colombiana de Investigación AgropecuariaAGROSAVIA y al Ministerio de Agricultura y Desarrollo Rural, por permitir el soporte logístico y financiero para el desarrollo de esta investigación, la cual se realizó mediante el Macroproyecto «Estrategias para mejorar la competitividad y sostenibilidad de la producción de carne y leche en el Caribe húmedo», administrado mediante el convenio $\mathrm{N} .^{\circ} 1828$.

\section{Literatura Citada}

1. [AOAC] Association of Official Agricultural Chemists Official Methods of Analysis. 2005. Official Methods of Analysis of AOAC International. $18^{\text {th }}$ ed. Gaithersburg, USA: AOAC.

2. Berman A. 2011. Are adaptations present to support dairy cattle productivity in warm climates? J Dairy Sci 94: 2147-2158. doi: 10.3168/jds.2010-3962

3. Carizi CHV, Garzón PJP, Alvarado MJP, Marini, PR. 2019. Condición corporal y su relación con producción láctea, reproducción y perfil metabólico en vacas lecheras del trópico boliviano. Rev Inv Vet Perú 30: 107-118. doi: 10.15381 /rivep. v30il.14459

4. Cervantes P, Trujillo S, Hernández A, Domínguez B, Andrade JA. 2014. Protein and endocrine homeostasis in dairy cows in tropical in transition period. AICA 4: 83-85.

5. Clariget JM, Quintans G, Banchero G, Alvarez-Oxiley A, Bentancur $O$, López-Mazz CR, Pérez-Clariget $R$. 2020. Effects of rice bran and glycerin supplementation on metabolic and productive responses of beef cows. $\mathrm{R}$ Bras Zootec 49:1-14. doi: 10.37496/ rbz4920190082

6. Claramunt M, Meikle A, Soca P. 2020. Metabolic hormones, grazing behaviour, offspring physical distance and productive response of beef cow grazing at two herbage allowances. Animal 14: 1520-1528. doi: 10.1017/S1751731119003021

7. De Lana FMF, Rennó NL, Detmann, E. Fonseca MP, de Campos VF, Moreira SS, Caio MH, et al. 2020. Performance, metabolic and hormonal responses of grazing Nellore cows to an energy-protein supplementation during the pre-partum phase. BMC Vet Res 16:113. doi: 10.1186/s12917-020-02309-3

8. Detmann E, Paulino MF, de Campos $V F$, Huhtanen P. 2014. Nutritional aspects applied to grazing cattle in the tropics: a review based on Brazilian results. Semina: Ciências Agrárias, 35: 2829-2854. doi: 10.5433/1679-0359.2014v35n4Suplp2829

9. Diskin MG, Kenny DA. 2016. Managing the reproductive performance of beef cows. Theriogenology 86: 379-387. doi:10.1016/j.theriogenology 2016.04.052

10. Fox DG, Tedeschi LO, Tylutki TP, Russell JB, Van Amburgh ME, Chase LE, Pell AN, Overton TR. 2004. The Cornell Net Carbohydrate and Protein System model for evaluating herd nutrition and nutrient excretion. Ani Feed Sci Tech 112: 29-78. doi: 10.1016/ j.anifeedsci.2003.10.006

11. Franco L, Calero D, Durán C. 2006. Manejo y utilización de forrajes tropicales multipropósitos. Palmira, Colombia: Univ. Nacional de Colombia. 30 p.

12. García GL, Orjuela AG, González FD, Rodríguez APR. 2019. Evaluación del estado metabólico, el peso vivo y la condición corporal durante la gestación en vacas de carne en la Altillanura colombiana. Orinoquia 23: 13-24. doi: 10.22579/20112629.565

13. Gross J, Van Dorland HA, Bruckmaier RM, Schwarz FJ. 2011. Performance and metabolic profile of dairy cows during a lactational and deliberately induced negative energy balance with subsequent realimentation. J Dairy Sci 94: 1820-1830. doi:10.3168/jds.2010-3707 
14. Hammeleers A. 2002. Métodos para estimar consumo voluntario de forrajes por rumiantes en pastoreo. [Internet]. Disponible en: https://n9.cl/f1s5v

15. [ICA] Instituto Colombiano Agropecuario. 2020. Censo poblacional bovino en Colombia 2020. [Internet]. Disponible en: https://www.ica.gov.co/areas/ pecuaria/servicios/epidemiologia-veterinaria/censos-2016/censo-2018.aspx

16. Klein JL, Adams SM, De Moura AF, Alves FDC, Maidana FM, Brondani IL, Cocco JM, et al. 2021. Productive performance of beef cows subjected to different nutritional levels in the third trimester of gestation. Animal 15: 1-6. doi: 10.1016/j.animal.2020.100089

17. Lopes SA, Paulino MF, Detmann E, Rennó LN, Valente ÉEL, Cabral CHA, Valério DCV, et al. 2016. Evaluation of grazing beef cows receiving supplements with different protein contents. Semina: Ciências Agrárias 37: 3361-3372. doi: 10.5433/ 1679-0359.2016v37n5p3361

18. Mapfumo L, Muchenje V, Mupangwa JF, Scholtz MM. 2017. Changes in biochemical proxy indicators for nutritional stress resilience from Boran and Nguni cows reared in dry arid rangeland. Trop Anim Health Prod 49: 1383-1392. doi:10.1007/s11250-017$1338-0$

19. Martínez-Rocha E, Ramírez R, Núñez R, García JG, Parra GM. 2020. Comportamiento de crecimiento y reproductivo en hembras bovinas Romosinuano. Rev MVZ Córdoba 26: 1- 9. doi: 10.21897/rmvz.2033

20. McArt JA, Nydam DV, Oetzel GR, Overton TR, Ospina PA. 2013. Elevated non-esterified fatty acids and $\beta$ hydroxybutyrate and their association with transition dairy cow performance. Vet J. 198:560-570. doi: 10.1016/ j.tvj1.2013.08.011

21. Melo MO, López PL, Melo MS. 2020. Diseño de experimentos: métodos y aplicaciones. En: Diseños completamente aleatorizados y tamaño de muestras. $2^{\circ}$ ed. Bogotá, Colombia: Univ. Nacional de Colombia. p 153-182.

22. Mestra VL, de Paula LR, da Silva JCP, Veloso CM., de Queiroz, AC, da Fonseca DM. Rennó LN. 2013. Desempenho de vacas mestiças em função de suplementação energética $\mathrm{e}$ proteica em dietas à base de cana-deaçúcar. RBAS 3:117-127. doi: 10.21206/ rbas.v3i1.198

23. Moore SM, DeVries TJ. 2020. Effect of diet-induced negative energy balance on the feeding behavior of dairy cows. $\mathrm{J}$ Dairy Sci 103: 7288-7301. doi: 10.3168/ jds.2019-17705

24. Moura FH, Costa TC, Trece AS, Melo LP, Manso MR, Paulino MF, Rennó $L N$, et al. 2020. Effects of energyprotein supplementation frequency on performance of primiparous grazing beef cows during pre and postpartum. AsianAustralas J Anim Sci 33:1430-1443. doi: 10.5713/ajas.19.0784

25. [NRC] National Research Council. 2000. Nutrient requirements of beef cattle. $7^{\text {th }}$ Rev. Ed: Washington, DC: National Academy Press.

26. Oliveira F, Toniollo GH, Oliveira AFD, Viu MA, Ferraz HT, Lopes DT, Gambarini ML. 2010. The effect of offering an energy and protein supplement to grazing Canchim beef cows either postpartum or both pre-and postpartum on lipid blood metabolites and folliculogenesis. Anim Reprod Sci 121: 39-45. doi: 10.1016/j.anireprosci.2010.04 .192

27. Ossa SG, David HA, Santana RM, Reza S, García PJ, Abuabara PY. 2013. Formación, desarrollo y caracterización fenotípica de los caracteres productivos y reproductivos del hato Romosinuano del banco de germoplasma de Colombia. Cienc Tec Agrop 14: 231-243. doi: 10.21930/rcta.vol14_num2_-art:503

28. Parra-Cortés R, Magaña MAM, Vázquez ATP. 2019. Intensificación sostenible de la ganadería bovina tropical ba- 
sada en recursos locales: alternativa de mitigación ambiental para América Latina. Revisión bibliográfica. Rev AIDA 115: 342-359.

29. Pfeifer LFM, Rodrigues WB, Nogueira E. 2021. Relationship between body condition score index and fertility in beef cows subjected to timed artificial insemination. Livestock Sci 248: 1-5. doi:10.1016/j.livsci.2021.104482

30. Piao DC, Wang T, Lee JS, Vega RS, Kang SK, ChoiYJ, Lee HG. 2015. Determination of reference intervals for metabolic profile of Hanwoo cows at early, middle and late gestation periods. J Ani Sci Biotech 6: 1-5. doi: 10.1186/ s40104-015-0009-0

31. Puppel K, Kuczyñska B. 2016. Metabolic profiles of cow's blood; a review. J Sci Food Agric 96: 4321-4328. doi: 10.1002/jsfa.7779

32. Quintela LA, Becerra JJ, Rey C, Díaz C, Cainzos J, Rivas F, Herradón PG 2011. Perfiles metabólicos en preparto, parto y postparto en vacas de raza $\mathrm{Ru}$ bia Gallega: estudio preliminar. Recursos Rurais 7: 5-14. doi:10.15304/rr.id88

33. Richards MW, Spitzer JC, Warner MB. 1986. Effect of varying levels of postpartum nutrition and body condition at calving on subsequent reproductive performance in beef cattle. J Anim Sci 62: 300-306. doi: 10.2527/jas 1986.622300x

34. Silveira MD, Restle J, Menezes LD, Brondani IL, Nörnberg JL, Callegaro AM. 2012. Metabólitos sanguíneos de vacas de corte suplementadas ou não com sais de cálcio de ácidos graxos du- rante o período pré e/ou pós-parto. Arq Bras Med Vet Zootec 64: 1418-1426. doi:10.1590/S0102-09352012000600003

35. Samadi F, Phillips NJ, Blache D, Martin GB, D'Occhio MJ. 2013. Interrelationships of nutrition, metabolic hormones and resumption of ovulation in multiparous suckled beef cows on subtropical pastures. Anim Reprod Sci 137:137-144. doi: 10.1016/j.anireprosci.2012.12.012

36. Sotelo D, Paulino MF, Rennó LN, Detmann E, Maza OR, Marquez DC, Soarez ML, de Almeida MD, Moura FH. 2018. Performance and metabolic status of grazing beef heifers receiving increasing protein supplementation preand postpartum. Anim Prod Sci 59: 12441252. doi:10.1071/AN17485

37. Wagner JJ, Lusby KS, Oltjen JW, Rakestraw J, Wettemann RP, Walters LE. 1988. Carcass composition in mature Hereford cows: estimation and effect on daily metabolizable. J Anim Sci 66:603-612. doi: 10.2527/jas1988.$663603 x$

38. Waterman RC, Butler WR. 2010. Metabolic signals of beef cow in negative energy balance. In: Proc IV Grazing Livestock Nutrition Conference. Colorado, USA.

39. Wittwer FM. 2000. Diagnóstico dos desequilibrios metabólicos de energía em rebanhos bovinos. En: Gonzáles F, Barcellos J, Ospina H, Ribeira L (eds). Perfil metabólico em rumiantes, seu uso em nutrição e doenças nutricionais. Porto Alegre, Brasil: Universidade Federal Do Rio Grande Do Sul. p 9-22. 